\title{
Systemic therapy of renal cell carcinoma
}

\author{
Viktor Grünwald $\cdot$ Alain Ravaud
}

Received: 30 November 2013/Accepted: 6 December 2013/Published online: 10 January 2014

(C) Springer-Verlag Berlin Heidelberg 2014

The first licensed targeted agents had access to the markets in Europe in 2006, which catalyzed a rapid development of such drugs in metastatic renal cell carcinoma (mRCC). In recent years, different tyrosine kinase inhibitors (TKI) were tested, exploiting the inhibition of neoangiogenesis in mRCC. Numerous studies later, the TKI hype is over and has ceased expectations regarding treatment outcome in mRCC.

In principle, inhibition of the mammalian target of rapamycin (mTOR) and the vascular endothelial growth factor receptor (VEGFR) represent the current backbone of therapy in mRCC. Current clinical research stretches beyond the scope of registrational trials and explores clinical questions, such as the best strategy for comorbid patients or those with specific sites of disease in order to individualize therapy.

The next steps in clinical development will require a better understanding of biology in $\mathrm{mRCC}$ and the development of novel druggable targets. The extension of target inhibition beyond the scope of VEGFR has led to the clinical development of 3rd generation TKIs of which cabozantinib is a putative candidate for second-line treatment.

Current clinical development for immunotherapeutics holds great promise in cancer. Vaccines and check-point inhibitors are currently explored in clinical trials and are likely to add another layer to the current backbone of therapies.

We therefore summoned current clinical questions in this topic issue on RCC, not only focusing on systemic therapies. Our aim is to provide a rational to weight the choice of therapeutic and diagnostic alternatives in clinical practice and deepen the insight into specific patient scenarios in $\mathrm{mRCC}$.

V. Grünwald ( $\square$ )

Hannover, Germany

e-mail: gruenwald.viktor@mh-hannover.de
A. Ravaud
Bordeaux, France 